\title{
Relationship between lung function and grip strength in older hospitalized patients: a pilot study
}

\author{
This article was published in the following Dove Press journal: \\ International Journal of COPD \\ 19 April 2017 \\ Number of times this article has been viewed
}

\author{
Sarah J Holmes' \\ Stephen C Allen ${ }^{2,3}$ \\ Helen C Roberts ${ }^{4,5}$ \\ 'Medicine and Elderly Care, \\ Hampshire Hospitals NHS \\ Foundation Trust, Winchester, \\ ${ }^{2}$ Medicine and Geriatrics, The \\ Royal Bournemouth Hospital \\ and Christchurch Hospitals NHS \\ Foundation Trust, Bournemouth, \\ ${ }^{3}$ Centre of Postgraduate Medical \\ Research and Education, Bournemouth \\ University, Poole, ${ }^{4}$ Academic \\ Geriatric Medicine, University of \\ Southampton, ${ }^{5}$ University Hospital \\ Southampton NHS Foundation Trust, \\ Southampton, UK
}

\begin{abstract}
Objective: Older people with reduced respiratory muscle strength may be misclassified as having COPD on the basis of spirometric results. We aimed to evaluate the relationship between lung function and grip strength in older hospitalized patients without known airways disease.
\end{abstract}

Methods: Patients in acute medical wards were recruited who were aged $\geq 70$ years; no history, symptoms, or signs of respiratory disease; Mini Mental State Examination $\geq 24$; willing and able to consent to participate; and able to perform hand grip and forced spirometry. Data including lung function (forced expiratory volume in 1 second $\left[\mathrm{FEV}_{1}\right]$, forced vital capacity [FVC], $\mathrm{FEV}_{1} /$ FVC, peak expiratory flow rate [PEFR], and slow vital capacity [SVC]), grip strength, age, weight, and height were recorded. Data were analyzed using descriptive statistics and linear regression unadjusted and adjusted (for age, height, and weight).

Results: A total of 50 patients ( 20 men) were recruited. Stronger grip strength in men was significantly associated with greater $\mathrm{FEV}_{1}$, but this was attenuated by adjustment for age, height, and weight. Significant positive associations were found in women between grip strength and both PEFR and SVC, both of which remained robust to adjustment.

Conclusion: The association between grip strength and PEFR and SVC may reflect stronger patients generating higher intrathoracic pressure at the start of spirometry and pushing harder against thoracic cage recoil at end-expiration. Conversely, patients with weaker grip strength had lower PEFR and SVC. These patients may be misclassified as having COPD on the basis of spirometric results.

Keywords: older, grip strength, spirometry, peak expiratory flow rate, slow vital capacity

\section{Introduction}

There is concern that older people with reduced respiratory muscle strength may be misclassified as having COPD on the basis of spirometric results and receive unnecessary and potentially harmful treatment. With increasing age, the smaller pulmonary airways more readily collapse and forced vital capacity (FVC), forced expiratory volume in 1 second $\left(\mathrm{FEV}_{1}\right)$, and forced expiratory flow decrease; there is an increase in functional residual capacity and expiratory reserve volume, residual volume increases, and there is little change in total lung capacity. ${ }^{1-3}$ In addition with aging, there is a decreased respiratory response to hypoxemia and hypercapnia and a reduced perception of raised resistance of the airway. ${ }^{2}$ There is a loss of synovial joints between the sternum and the costal cartilages reducing chest wall compliance and tension forces, leading to reduced maximal respiratory pressure and decreased muscle mass and strength of accessory respiratory muscles. ${ }^{4}$ It has also been observed that there is a significant decrease by $25 \%$ in the diaphragm strength in the older compared with young adults. ${ }^{5}$ One study has observed that inspiratory (mainly diaphragmatic)
Correspondence: Sarah J Holmes Medicine and Elderly Care, Hampshire Hospitals NHS Foundation Trust, Royal Hampshire County Hospital, Romsey Road, Winchester SO22 5DG, UK Emailsarah.holmes@hhft.nhs.uk 
strength has been considered to be the most relevant in relation to function, fatigue, and training. ${ }^{6}$

With aging, there is a triad of loss of muscle mass, strength, and function defined as sarcopenia. This is common and has serious consequences including increased risk of current and future falls, dependency, hospitalization, and mortality. Grip strength is recommended as a simple standardized clinical measure used in the diagnosis of sarcopenia. $^{7}$ Age-associated skeletal muscle changes may impact respiratory muscle function, and a positive correlation between grip strength and inspiratory and expiratory muscle strength has been demonstrated..$^{8-10}$ Reduced lung function (in particular $\mathrm{FEV}_{1}$ and $\mathrm{FVC}$ ) after adjusting for age, smoking, and physical activity has been demonstrated with reduced skeletal muscle mass in Korea. ${ }^{11}$

COPD is defined by the Global initiative for chronic Obstructive Lung Disease (GOLD) by symptoms, exposure to risk factors (namely smoking), family history, and confirmation with spirometry with a postbronchodilator $\mathrm{FEV}_{1}$ / $\mathrm{FVC}<0.70$ and postbronchodilator $\mathrm{FEV}_{1}$ (to assess degree of airflow limitation). ${ }^{12}$

Discord over the standardized definition of COPD across international groups (whether a fixed ratio, use of standardized age-related reference spirometry ranges, or lower limit than normal spirometry values) makes it difficult to compare the prevalence of COPD across populations and across studies due to the lack of standardized protocol. ${ }^{13} \mathrm{FEV}_{1} / \mathrm{FVC}$ ratio decreases with aging and is included in the GOLD definition of COPD using the criteria of postbronchodilator $\mathrm{FEV}_{1} / \mathrm{FVC}<0.70$. There is a decrease in $\mathrm{FEV}_{1}$ with aging likely to be due to age-related airway compliance changes and this is incorporated in the GOLD definition to assess the severity of airflow limitation. Both criteria have potential to lead to increasing diagnosis of COPD in older nonsmoking patients with no lung pathology. ${ }^{14}$ Normal reference spirometry values have been defined in older age groups; however, these are not included in the international definition of COPD. ${ }^{15,16}$

Peak expiratory flow rate (PEFR) in older adults is an inexpensive measure, assessed alongside other respiratory function and used in previous studies evaluating grip strength, respiratory muscle strength, and lung function. ${ }^{17}$ It measures maximal expiratory flow, is effort dependent, and represents airflow through larger caliber airways. ${ }^{18}$ PEFR has been used to assess adherence and benefit of treatments for airways disease and one of its limitation is the variability in performance in older persons. Older age, reduced body size, smoking, cardiovascular, respiratory, and cognitive diseases are associated with a reduced PEFR. ${ }^{17,19}$
Slow vital capacity (SVC) may be easier for some older adults who have difficulty coordinating the more FVC due to factors, such as reduced cognitive ability, coughing during the maneuver, weakness, or fatiguability. ${ }^{20}$ Again SVC is an inexpensive measure that can be tested alongside other respiratory measures using the same spirometry equipment. However, it is not incorporated in the international definitions of airways obstruction. It is also recognized that some older adults with cognitive impairment may be unable to perform both SVC and FVC. ${ }^{20}$

There is increasing interest in the relationship between sarcopenia, respiratory muscle strength, and lung function in patients with COPD. ${ }^{21-23}$ However, there has been very limited evaluation of sarcopenia, respiratory muscle strength, and lung function in older people without known airways disease. This study aimed to evaluate the relationship of grip strength and lung function in older hospital patients without known respiratory disease.

There is an increasing recognition of an association between muscle strength and lung function in people with COPD. It is unknown whether there is a relationship between grip strength and lung function in older non-smoking adults. In this study, older non-smoking patients with weaker grip strength had lower PEFR and SVC. Reduced spirometric lung function measurements in weaker older individuals may contribute to misdiagnosis of COPD.

\section{Materials and methods \\ Study design and ethics}

This was a prospective study on patients aged $\geq 70$ years admitted to acute older people's wards at a university hospital in the UK over a 6-month period. Southampton and South West Hampshire Research Ethics Committee gave approval for this study, which was registered with ClinicalTrials.gov (NCTO1647204).

\section{Participants}

The inclusion criteria for recruitment were age $>70$ years, a history of never smoking or trivial smoking $<1$ year, no history, symptoms or signs of respiratory disease, and negative for bronchial obstruction on the respiratory symptom questionnaire. ${ }^{24}$

Exclusion criteria were met if any participant was unable to meet all of the inclusion criteria, had a Mini Mental State Examination (MMSE) score of $<24$, or had any contraindication to performing spirometry according to the Association for Respiratory Technology and Physiology (ARTP) guidelines. ${ }^{25}$ These contraindications included hemoptysis; pneumothorax; unstable angina; myocardial 
infarction in the last one month; pulmonary embolism; thoracic, abdominal, or cerebral aneurysms; recent eye surgery; nausea or vomiting; and recent thoracic or abdominal surgery. In addition, if participants appeared nonspecifically unwell or nursing staff had concerns about participation, then they were not approached.

A convenience sample of eligible patients (based on the researcher's availability) were approached for recruitment, then given a patient information sheet and opportunity to ask further questions before written informed consent was obtained. Each participant was taken through the brief respiratory questionnaire to ensure they had no symptoms that would affect carrying out the spirometry or exclude them from the study: any positive response to the questionnaire excluded recruitment to the study. Participants were also asked about any contraindication to performing spirometry according to the ARTP guidelines, and this was confirmed on review of case notes before performing spirometry with the participants' permission.

\section{Data collection}

Participants' height was calculated from ulna length measurement, ${ }^{26}$ as the majority of participants were unable to stand. Weight was measured using sitting scales at the bedside. Body mass index was calculated by dividing body weight by the square of height $\left(\mathrm{kg} / \mathrm{m}^{2}\right)$. Cognitive function was assessed using the MMSE, ${ }^{27}$ which has been an established screening tool in spirometry studies in older people.

A calibrated Jamar dynamometer was used to measure grip strength using a standard protocol. Three measurements were recorded for each hand to the nearest $1 \mathrm{~kg}$ and the maximum grip recorded. ${ }^{28}$ A Microlab Portable spirometer was used to measure $\mathrm{FEV}_{1}$, FVC, PEFR (best of five measurements recorded), and SVC (best of three measurements recorded). The spirometer was calibrated for each assessment. Measurements were recorded in the sitting position, feet on the floor, spine erect, and without support for the upper limbs. Repeatability was considered to be adequate when there was a difference of $\leq 0.15 \mathrm{~L}$ between the maximum and the next largest FVC and between the maximum and next largest $\mathrm{FEV}_{1}$. In patients with $\mathrm{FVC} \leq 1 \mathrm{~L}$, a difference of $\leq 0.10 \mathrm{~L}$ was used. SVC attempts were considered to be acceptable if the individual breathed in to total lung capacity then breathed out until flow had ended for at least 1 second. SVC measurements were considered to be repeatable if the two highest attempts varied by $\leq 0.2 \mathrm{~L}$. The highest recording was taken for each measurement. However, to assure the quality of these data, two observers reviewed spirograms of each patient separately and together to see if they reflected adequate performance. ${ }^{29}$

\section{Statistical analysis}

Data were entered into a database and analyzed using IBM SPSS Statistics 20 software separately for men and women. Normality of variables was assessed using visual inspection of histograms and skewness. Mean and standard deviation, median and interquartile ranges were used to describe the participants' demographic and clinical characteristics, grip strength, and spirometry results. Comparison between men and women was conducted using independent sample $t$-tests and the Mann-Whitney $U$-test accordingly. The association between lung function variables and grip strength were assessed for men and women separately using linear regression analysis both unadjusted and adjusted for age, height, and weight.

\section{Results}

A total of 601 patients were screened over 6 months of which 509 were excluded: 58 patients with acute respiratory illness, 48 with known respiratory disease, 109 patients with more than 1 year of smoking history, 13 with acute illness, and 281 with known moderate or severe cognitive impairment. The 92 eligible patients were approached with information leaflets and of these 50 (54\%) consented to take part in the study (Figure 1).

Patient characteristics are recorded in Table 1. Spirometry was difficult to achieve reproducibility of the results and for patients to rehearse the technique without becoming fatigued, to ensure effort in the early part of forced expiration, and to avoid patients stopping mid-expiration. After review of 10 patients, SVC was introduced for the remaining 40 patients. SVC can be easier and more relaxed than FVC, and can avoid coughing triggered by the forced maneuver. ${ }^{20}$

The association of lung function with grip strength is shown in Table 2. There was a wide variation in participants'

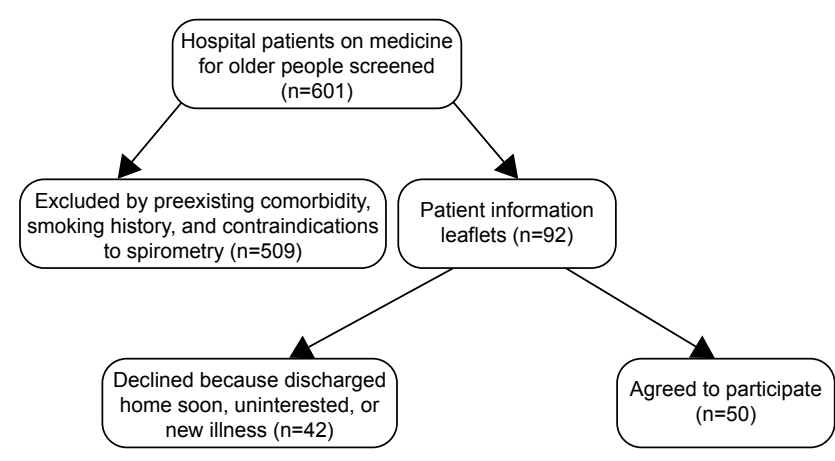

Figure I Flowchart for the recruitment into the study. 
Table I Participant characteristics and their grip strength and spirometry

\begin{tabular}{|c|c|c|c|}
\hline Characteristics & $\begin{array}{l}\text { Men } \\
(n=20)\end{array}$ & $\begin{array}{l}\text { Women } \\
(n=30)\end{array}$ & $P$-value \\
\hline Age, years ${ }^{a}(n=50)$ & $86.3(4.9)$ & $87.5(4.8)$ & 0.44 \\
\hline Height, $m^{a}(n=50)$ & I.7| (0.7) & $\mathrm{I} .60(0.7)$ & 0.81 \\
\hline Weight, kg $(n=50)$ & $74.9(15.1)$ & $66.0(16.4)$ & 0.64 \\
\hline BMI, $\mathrm{kg} / \mathrm{m}^{2 \mathrm{a}}(\mathrm{n}=50)$ & $25.6(5.3)$ & $26.0(6.4)$ & 0.36 \\
\hline $\operatorname{MMSE}^{\mathrm{b}}(\mathrm{n}=50)$ & $26(24,28)$ & $27(25,29)$ & 0.04 \\
\hline Grip strength, $\mathrm{kg}^{\mathrm{a}}(\mathrm{n}=50)$ & $19.5(7.21)$ & I2.4 (3.73) & 0.03 \\
\hline $\mathrm{FEV}_{1}, \mathrm{~L} / \mathrm{s}^{\mathrm{c}}(\mathrm{n}=50)$ & $1.7(0.5)$ & $1.0(0.3)$ & 0.02 \\
\hline FVC, $L^{c}(n=50)$ & $2.1(0.7)$ & $\mathrm{I} .4(0.4)$ & 0.01 \\
\hline $\mathrm{FEV}_{1} / \mathrm{FVC}, \%^{\mathrm{c}}(\mathrm{n}=50)$ & $77.6(14.3)$ & $73.6(12.2)$ & 0.88 \\
\hline PEFR, $L^{c}(n=50)$ & $262.1(102.5)$ & |48.| (57.5) & 0.004 \\
\hline SVC, $L^{c}(n=40)$ & $2.1(0.9)$ & $1.2(0.4)$ & 0.003 \\
\hline
\end{tabular}

Notes: ${ }^{a}$ Mean (SD); ${ }^{\mathrm{b}}$ median (IQR); ' $\mathrm{cmean}(\mathrm{SD})$ using $t$-test. $P$-values were calculated using Student's $t$-test.

Abbreviations: $\mathrm{BMI}$, body mass index; $\mathrm{FEV}$, forced expiratory volume in I second; FVC, forced vital capacity; MMSE, Mini Mental State Examination; PEFR, peak expiratory flow rate; SVC, slow vital capacity; SD, standard deviation; IQR, interquartile range.

PEFR values. In men, grip strength was significantly associated with $\mathrm{FEV}_{1}$ but this was attenuated by adjustment for age, height, and weight. Significant associations were found in women between grip strength and PEFR and between grip strength and SVC, both of which remained robust to adjustment. No other significant relationship was found.

\section{Discussion}

Grip strength was significantly stronger, and lung function was significantly larger in men compared with women probably due to their larger body size allowing them to generate greater intrathoracic pressures and higher lung volumes. Stronger grip strength was significantly associated with a larger $\mathrm{FEV}_{1}$ in men, but the significance was attenuated after adjustment for age, height, and weight.

No significant relationship was found between grip strength and $\mathrm{FEV}_{1}$ in women. In line with previous reports, this study demonstrated variation in PEFR values among the participants. ${ }^{17-19}$ Reduced PEFR was demonstrated in women with low grip strength. Low $\mathrm{FEV}_{1}$ and low PEFR in weaker individuals could be due to increased parenchymal compliance. PEFR may be a useful measure of airflow obstruction, and change in response to treatment may be less reliable in older women with low grip strength.

Increased grip strength was associated with a larger FVC in both men and women but was not statistically significant. There was no significant association between grip strength and ratio of $\mathrm{FEV}_{1} / \mathrm{FVC}$. This result reflected patients with a similar reduction in both $\mathrm{FEV}_{1}$ and $\mathrm{FVC}$ and a lack of consistency in spirometry. Importantly, SVC appeared to be a more practical and applicable lung function measure in this older patient group than FVC.

Stronger grip strength was significantly associated with larger PEFR and SVC in women but not in men. PEFR and SVC may be more significant in women in this study reflecting stronger patients generating higher intrathoracic pressure at the start of spirometry and pushing harder against thoracic cage recoil at end-expiration. Differences found between women and men may be related to smaller airways and lung volumes in women compared with men.

A relationship between grip strength and $\mathrm{FEV}_{1}$ in patients who were healthy subjects and with COPD was inferred in a recent study in India. This study demonstrated that a decrease in grip strength was associated with a decrease in $\mathrm{FEV}_{1}$ in women and in FVC in men; however, it did not adjust for age or body size. ${ }^{30}$ Current studies that report $\mathrm{FEV}_{1} / \mathrm{FVC}$ do so only in the context of patients with lung pathology, smokers, or a mixed group and do not compare this to grip strength or adjust for body size. ${ }^{31}$ One study showed a significant correlation between grip strength and PEFR in hospital staff but again did not adjust for age and height. ${ }^{17}$ A further limitation of current research in the relationship between grip strength and lung function is a lack of standardization of instruments used and methodology particularly in assessing older subjects. There is also a lack of consensus

Table 2 Association of grip strength and lung function in the older participants

\begin{tabular}{|c|c|c|c|c|c|c|c|c|}
\hline \multirow[t]{3}{*}{ Lung function } & \multicolumn{4}{|l|}{ Men } & \multicolumn{4}{|c|}{ Women } \\
\hline & \multicolumn{2}{|c|}{ Unadjusted } & \multicolumn{2}{|c|}{ Adjusted $^{\mathrm{a}}$} & \multicolumn{2}{|c|}{ Unadjusted } & \multicolumn{2}{|c|}{ Adjusted $^{a}$} \\
\hline & $\beta$ & $P$ & $\beta$ & $P$ & $\beta$ & $P$ & $\beta$ & $P$ \\
\hline $\mathrm{FEV}_{1}(\mathrm{~L} / \mathrm{s})$ & 0.03 & 0.05 & 0.04 & 0.06 & 0.02 & 0.24 & 0.02 & 0.27 \\
\hline FVC (L) & 0.04 & 0.07 & 0.06 & 0.07 & 0.02 & 0.33 & 0.02 & 0.35 \\
\hline FEV,/FVC (\%) & 0.08 & 0.87 & -0.14 & 0.85 & 0.17 & 0.79 & 0.17 & 0.79 \\
\hline PEFR (L) & 4.61 & 0.16 & 6.60 & 0.15 & 6.88 & 0.01 & 6.94 & 0.02 \\
\hline SVC (L) & 0.03 & 0.25 & 0.04 & 0.34 & 0.05 & 0.03 & 0.05 & 0.05 \\
\hline
\end{tabular}

Note: ${ }^{a}$ Adjusted results for age, height, and weight.

Abbreviations: $\beta$, Beta coefficient; FEV , forced expiratory volume in I second; FVC, forced vital capacity; PEFR, peak expiratory flow rate; $P$, probability value calculated using SPSS; SVC, slow vital capacity. 
on appropriate spirometry measures and protocols within research and agreement as to defined spirometry measures in clinical practice for diagnosing COPD.

There were limitations to the study. The sample size was small and patients with cognitive impairment, smokers $>1$ year, or acutely ill were excluded. The significance of this study's findings has to be taken within this context and are preliminary findings. Recruiting participants based on the researcher's availability may have led to potential eligible patients not being included but would not have resulted in observer bias.

This study examined individuals far older than in previous studies and this older group had difficulty maintaining the consistency to conform to the American Thoracic Society (ATS) guidelines for spirometry. We reported the maximum spirometry values rather than the mean values recognizing that with repeated testing some patients may fatigue. This could be standardized by setting limits to exclude lung function tests, for example, with $\mathrm{FEV}_{1} / \mathrm{FVC}>90 \%$ or $<60 \%$ and abnormal spirogram patterns. Assessment of SVC in all participants would have been beneficial; however, this was not possible to obtain due to participants being discharged home. Further evaluation of SVC to measure airway obstruction particularly with those limited by coughing may be useful as a research tool and in practice.

There were also several strengths to this study. We performed it with a single researcher with trained competency in collecting data confirmed with intraobserver variability assessment. The study was carried out using calibrated instruments. The use of a standardized method for measuring grip strength was advantageous and is not usually the case in other studies in older persons. Similarly, we performed spirometry in a standardized way that would allow easy comparison to other studies.

We considered the appropriateness of performing spirometry in hospitalized patients given the potential instability of this population. Previous studies have highlighted variability in spirometry results particularly in older participants with cognitive impairment (due to an inability to coordinate or perform the test in a standardized manner), those acutely ill, and smokers (secondary to pathophysiologic changes). ${ }^{20}$ Therefore, we decided to use rigorous exclusion criteria to standardize recruitment and enable comparison with other studies, although we acknowledge its limitation on sample size.

This study aimed to evaluate the relationship between grip strength and lung function in older persons and whether current guidelines may inadvertently lead to clinicians misdiagnosing and potentially treating older nonsmokers with unnecessary and potentially harmful treatment. A larger community-based study using the same rigorous methodology may provide a better understanding of this relationship.

It could be that a larger number of older persons in the community would benefit from this research due to the increasing screening programs in primary care. It may also be easier to perform grip strength and spirometry within an outpatient setting in a more consistent fashion. It has also been demonstrated in older adults that fixed ratio $\mathrm{FEV}_{1} / \mathrm{FVC}$ may better identify subjects at risk of death or hospitalization than using the lower limit of normal spirometry values. ${ }^{32}$ Hence, this area of debate may be more complicated than simply disease classification.

Future work may consider review of grip strength to lung function expressed in terms of the diagnostic cutoff values for COPD using both the lower limit of normal and the fixed ratio criteria.

There may be other avenues of assessing the relationship between grip strength and spirometry in older nonsmokers using additional biochemical markers for aging and measuring FEV at the earlier part of forced expiration to increase accuracy of results before fatigability sets in.

Pulmonary rehabilitation programs are established for patients with COPD. Similarly resistance training has been recognized as important in maintaining muscle mass and strength in older people. There may be the potential for pulmonary rehabilitation programs for older people in the future to maintain respiratory muscle mass and strength. ${ }^{32}$

\section{Conclusion}

Men had significantly stronger grip strength and larger $\mathrm{FEV}_{1}$, FVC, PEFR, and SVC compared with women probably because of larger body size. There was a significant association between grip strength and PEFR and grip strength and SVC after adjustment for age, height, and weight in women, which may reflect stronger patients generating higher intrathoracic pressure at the start of spirometry and pushing harder against thoracic cage recoil at end-expiration. In men, a significant relationship was found between grip strength and $\mathrm{FEV}_{1}$, which was attenuated after adjustment for age, height, and weight, and this requires further evaluation.

\section{Acknowledgments}

SJ Holmes was supported by a British Geriatric Society start-up grant. This study was supported by the National Institute for Health Research (NIHR) Southampton Biomedical Research 
Centre in Nutrition, the MRC Lifecourse Epidemiology Unit, the University of Southampton and the British Geriatrics Society. HC Roberts is supported by the NIHR Collaboration for Leadership in Applied Health Research and Care (CLAHRC): Wessex. This report is independent research by the BRC and CLAHRC: Wessex. The views expressed in this publication are those of the author(s) and not necessarily those of the NHS, the National Institute for Health Research, or the Department of Health.

\section{Disclosure}

The authors report no conflicts of interest in this work.

\section{References}

1. Britto RR, Vieira DS, Botoni FA, Botoni ALAS, Velloso M. The presentation of respiratory failure in elderly individuals. Curr Geriatr Rep. 2015;4(2):166-173.

2. Janssens JP, Pache JC, Nicod LP. Physiological changes in respiratory function associated with ageing. Eur Respir J. 1999;13(1):197-205.

3. Pride NB. Ageing and changes in lung mechanics. Eur Respir J. 2005; 26(4):563-565.

4. Janssens JP. Aging of the respiratory system: impact on pulmonary function tests and adaptation to exertion. Clin Chest Med. 2005;26(3): 469-484.

5. Tolep K, Higgins N, Muza S, Criner G, Kelsen SG. Comparison of diaphragm strength between healthy adult elderly and young men. Am Journal Respiratory and Critical Care Medicine. 1995;152(2):677-682.

6. Kim MJ. Respiratory muscle training: implications for patient care. Heart Lung. 1984;13(4):333-340.

7. Cruz-Jentoft AJ, Baeyens JP, Bauer JM, et al. Sarcopenia: European consensus on definition and diagnosis: report of the European working group on sarcopenia in older people. Age Ageing. 2010;39(4): $412-423$.

8. Summerhill EM, Angov N, Garber C, McCool FD. Respiratory muscle strength in the physically active elderly. Lung. 2007;185(6):315-320.

9. Doherty TJ. Invited review: aging and sarcopenia. J Appl Physiol. 2003; 95(4):1717-1727.

10. Polla B, D'Antona G, Bottinelli R, Reggiani C. Respiratory muscle fibres: specialisation and plasticity. Thorax. 2004;59(9):808-817.

11. Jeon Y, Shin M, Kim M. Low pulmonary function is related with a high risk of sarcopenia in community-dwelling older adults: the Korea national health and nutrition examination survey (KNHANES) 2008-2011. Osteoporos Int. 2015;1-7.

12. Global Initiative for Chronic Obstructive Lung Disease (GOLD). Global strategy for the diagnosis, management, and prevention of chronic obstructive pulmonary disease. 2015. Available from: http://www. goldcopd.org. Accessed August 14, 2016.

13. Swanney MP, Ruppel G, Enright PL, et al. Using the lower limit of normal for the FEV1/FVC ratio reduces the misclassification of airway obstruction. Thorax BMJ. 2008;63:1046-1051.
14. Hardie JA, Buist AS, Vollmer WM, Ellingsen I, Bakke PS, Mørkve O. Risk of over-diagnosis of COPD in asymptomatic elderly never-smokers. Eur Respir J. 2002;20(5):1117-1122.

15. Crapo RO, Morris AH, Gardner RM. Reference spirometric values using techniques and equipment that meet ATS recommendations. Am Rev Respir Dis. 1981;123:659-664.

16. Enright PL, Kronmal RA, Higgins M, Schenker M, Haponik EF. Spirometry reference values for women and men 65 to 85 years of age. Cardiovascular health study. Am Rev Respir Dis. 1993;147:125-133.

17. Hornby ST, Nunes QM, Hillman TE, et al. Relationships between structural and functional measures of nutritional status in a normally nourished population. Clin Nutr. 2005;24:421-426.

18. Hegewald MJ, Lefor MJ, Jensen RL, et al. Peak expiratory flow is not a quality indicator: peak expiratory flow variability and FEV1 are poorly correlated in an elderly population. Chest. 2007;131(5):1494-1499.

19. Cook NR, Albert MS, Berkman LF, Blazer D, Taylor JO, Hennekens CH. Interrelationships of peak expiratory flow rate with physical and cognitive function in the elderly: MacArthur Foundation Studies of Aging. $J$ Gerontol A Biol Sci Med Sci. 1995;50(6):M317-M323.

20. Allen SC, Charlton C, Backen W, Warwick-Sanders M, Yeung P. Performing slow vital capacity in older people with and without cognitive impairment-is it useful? Age Ageing. 2010;35(9):588-591.

21. Jones SE, Maddock M, Kon SS, et al. Sarcopenia in COPD: prevalence, clinical correlates and response to pulmonary rehabilitation. Thorax. 2015; 70:213-218.

22. Orozco-Levi M. Structure and function of the respiratory muscles in patients with COPD: impairment or adaptation? Eur Respir J. 2003; 22(46):41s-51s.

23. Heunks LM, Dekhuiizen PN. Respiratory muscle function and free radicals: from cell to COPD. Thorax. 2000;55:704-716.

24. Venables KM, Farrer N, Sharp L, Graneek BJ, Newman Taylor AJ. Respiratory symptoms questionnaire for asthma epidemiology: validity and reproducibility. Thorax. 1993;48:214-219.

25. Cooper BG. An update on contraindications for lung function testing. Thorax. 2011;66(8):714-723.

26. Malnutrition Advisory Group. Alternative measurements: instructions and tables, British Association for Parenteral and Enteral Nutrition. 2013. Available from: www.bapen.org.uk. Accessed August 12, 2016.

27. Folstein MF, Folstein SE, McHugh PR. Mini-Mental state: a practical method for grading the cognitive state of patients for the clinician. J Psychiatr Res. 1975;12(3):189-198

28. Roberts HC, Denison HJ, Martin HJ, et al. A review of the measurement of grip strength in clinical and epidemiological studies: towards a standardised approach. Age Ageing. 2011;40(4):423-429.

29. Miller MR, Hankinson J, Brusasco V, et al. Standardisation of spirometry. Eur Respir J. 2005;26(2):319-338.

30. Shah S, Nahar P, Vaidya S, Salvi S. Upper limb muscle strength and endurance in chronic obstructive pulmonary disease. Indian J Med Res. 2013;138(4):492-496.

31. Ozden O, Zeynep E, Kokturk N, Karatas GK. Hand grip strength in patients engaged in pulmonary rehabilitation program during COPD exacerbation. Eur Respir J. 2011;38(6 Suppl 55):1247.

32. Quanjer PH. Diagnosing COPD: high time for a paradigm shift. Respir Care. 2011;56(11):1861-1863.
International Journal of COPD

\section{Publish your work in this journal}

The International Journal of COPD is an international, peer-reviewed journal of therapeutics and pharmacology focusing on concise rapid reporting of clinical studies and reviews in COPD. Special focus is given to the pathophysiological processes underlying the disease, intervention programs, patient focused education, and self management protocols.

\section{Dovepress}

This journal is indexed on PubMed Central, MedLine and CAS. The manuscript management system is completely online and includes a very quick and fair peer-review system, which is all easy to use. Visit http://www.dovepress.com/testimonials.php to read real quotes from published authors. 